\title{
Supernova SN 2008iz in M82 Galaxy
}

\author{
N. Kimani ${ }^{1}$, A. Brunthaler ${ }^{1}$, K. M. Menten $^{1}$, I. Martí-vida ${ }^{12}$ \\ ${ }^{1}$ Max-Planck-Institut fǔr Radioastronomie, Auf dem Hǔgel 69, 53121 Bonn, Germany, nkimani@mpifr- \\ bonn.mpg.de \\ ${ }^{2}$ Onsala Space Observatory, Chalmers University of technology, SE-43992, Onsala (Sweden)
}

\begin{abstract}
We report on multi-frequency Very Large Array (VLA) radio observations for an on-going monitoring campaign of supernova SN 2008iz in the nearby galaxy M82. We fit two light curve models to the data, a simple power-law model and a simplified Weiler model, yielding a decline index, $\beta=-1.23 \pm 0.01$ and $-1.41 \pm 0.02$, respectively. The late time radio light curve evolution shows $a$ flux density enhancement at $\sim 1000$ and $\sim 1700$ days which are a factor of $\sim 2$ and $\sim 4$ higher than the surrounding flux, respectively. The flux density enhancement from day 1700 does not show signs of decline from results obtained so far. The enhancement is attributed to SN 2008iz expanding shock wave encounter with a clumpy dense circumstellar medium.
\end{abstract}

Keywords: Supernova, SN 2008iz, Radio continuum, M82 galaxy

\section{Introduction}

The Radio supernova SN 2008iz was discovered in M82 galaxy which is a nearby irregular (IO) galaxy forming part of M81 group at a distance of $3.63 \mathrm{Mpc}$ (Freedman et al. 1994). Radio supernove are supernovae that are visible at radio wavelengths. They are rare events with just a few dozens detected so far (Weiler et al. 2002). The lack of detections is attributed to either the supernovae being relatively distance or fairly weak, making them difficult to study in details. So far, SN 1993J in M81 is the best studied radio supernova, due to its proximity $(3.63 \mathrm{Mpc})$, unobscured environment and face-on galaxy orientation (Marcaide et al. 1997, Bietenholz et al. 2010a, Martí-vidal et al. 2011a,b). The more recent discovery of radio supernova SN 2008iz (Brunthaler et al. 2009a) offers the possibility to study another supernova at a similar distance in great details and make comparisons to SN 1993J.

The supernova SN 2008iz was discovered by Brunthaler and his collaborators on April 2009 using the Very Large Array (VLA) at 22 GHz (Brunthaler et al. 2009a). The discovery was confirmed with Multi-Element Radio Linked Interferometer Network (MERLIN) at $5 \mathrm{GHz}$ (Muxlow et al. 2009; Beswick et al. 2009) and Urumqi single dish telescope at $5 \mathrm{GHz}$ (Marchili et al. 2010). The endeavours to make detections in other astronomical windows were not successful. For instance, there are no detections in visible light, Near-IR (Fraser et al. 2009) and X-Ray (Brunthaler et al. 2009b). The non-detection at other astronomical windows indicate that the supernova explosion happened behind a large gas or dust cloud, and make it 
hard to classify the supernova. However, since type Ia supernova are not known to show strong radio emission, SN 2008iz is most likely a core collapse supernova i.e. either Type Ib/c or Type II (Brunthaler et al. 2009b).

SN 2008iz is located in M82 galaxy at RA $09^{\mathrm{h}} 55^{\mathrm{m}} 51^{\mathrm{s}} .551$ and DEC $69^{\circ} 40^{\prime} 45^{\prime \prime} .792$ (J2000, Brunthaler at al. 2009b). This position is 2.5" (43 pc assuming a distance of $3.6 \mathrm{Mpc}$ ) SW of the photometric center of M82 based on $2.2 \mu \mathrm{m}$ peak (Weiß et al. 2001). From their monitoring projects, it was found that the supernova explosion date is estimated to be 18 Feb $2008 \pm 6$ days (Marchili et al. 2010).

The VLBI study reveals the expansion velocity of SN 2008iz to be approximately $21,000 \mathrm{kms}^{-1}$ making it one of the fastest expanding radio supernova and also show a self-similar behaviour (Brunthaler et al. 2010). The self-similarity describes that the source shows similar structure on all scales when scaled to the same resolution. The derived spectral index in radio is $\alpha=-1.08 \pm 0.08$ (Brunthaler et al. 2010) and the deceleration index $(m)$, which indicates that the deceleration of the supernova is 0.89 (Brunthaler et al. 2010, Marchili et al. 2010). Both Brunthaler et al (2010) and Marchili et al. (2010), find that synchrotron self-absorption (SSA) is not important when modelling SN 2008iz.

Multi-frequency measurements of the radio light-curves and their time dependent evolution help in understanding important information such as the density and structure of the circumstellear medium (CSM), evidence for possible binary companions, clumpiness or filamentation in the pre-supernova wind, and presupernova mass loss rates. We present a multi-frequency VLA evolution results of SN 2008iz for a period of 7yrs since its explosion. We also combine our observations with other archival data to get a comprehensive picture of its radio light curve evolution. In this paper, we describe the monitoring and data reduction process in section 2 . In section 3 we present the multi-frequency light-curve results. In section 4 we discuss the results while in section 5, we present a brief summary.

\section{Observations and Data calibration}

The radio monitoring campaign was conducted with the VLA, at $\mathrm{L}(1.4 \mathrm{GHz}), \mathrm{C}$ $(4.8 \mathrm{GHz}), \mathrm{X}(8.4 \mathrm{GHz}), \mathrm{K}(22.3 \mathrm{GHz})$ and Q-bands $(43.2 \mathrm{GHz})$ to trace the evolution of the radio emission from supernova SN 2008iz since its discovery. The observation was done in the standard continuum observation mode with a total bandwidth of $128 \mathrm{MHz}$, each in dual circular polarization. Flux density measurements were derived using calibrator 3C48 and phase referenced against calibrator J1048+7143. The flux calibrator was observed for a total time of 2 minutes in each observation. The observation used a switching cycle of six minutes, spending on average 1 minute on the phase calibrator and 5 minutes on M82. The cycles were repeated 5 times over the observation, yielding an integration time of $\square 25$ minutes on M82 at each frequency.

The last epoch of VLA observations on 23 Jan 2014 is an observation done in search of a newly discovered supernova $\mathrm{SN} 2014 \mathrm{j}$ at $\mathrm{C}$ and K-bands in 
configuration $\mathrm{AB}$. The data was taken in the standard continuum observation mode with a total bandwidth of $128 \mathrm{MHz}$, each in dual circular polarization. The flux calibrator 3C48 was observed for a total time of 3 minutes. The observation used a switching cycle of 9 minutes, spending an average time of 30 seconds on the phase calibrator J1048+7143 and 8 minutes on M82. The cycle was repeated 4 times over the observation, yielding an integration time of approximately 32 minutes on M82 at both frequencies.

\section{Results}

The standard data reduction steps and packages in AIPS are used in data reduction and calibration process. Inorder to reduce the contribution of galactic continuum flux emission to the SN 2008iz flux density, we restrict the interferometric $(\mathrm{u}, \mathrm{v})$ distance to $>30 \mathrm{k} \lambda$. The AIPS task 'JMFIT' is used to determine the flux density by fitting a 2D Gaussian fit to the visually identified compact SN 2008iz source. The flux density errors are derived by adding in quadrature the formal errors from the 2D Gaussian fit, a 5\% uncertainty error due to continuum interference, and the difference between peak and integrated flux values. The 'GNUPLOT' package is used to fit the results using equations 1 and 2 .

\section{Discussion}

The light curve of the optically-thin regime of SN 2008iz is well fit by a power-law of the form shown by equation 1 , especially at $22.3 \mathrm{GHz}$ which is our best sampled observation (see Fig. 1)

$$
S=K_{0}\left(t-t_{0}\right)^{\beta}
$$

where $\mathrm{K}_{0}$ is a scaling factor, $\mathrm{t}$ is the number of days since the explosion, $\mathrm{t}_{0}$ is the explosion day (18 Feb 2008), and the power-law decline index, $\beta$, which describes the observed flux density decline in the optically-thin regime at a later evolution date. The parameter $\beta$ is often affected by both the spectral index, $\alpha$, and the opacity $\tau$, an indication of high environmental dependence. Using our data, we fit a single $\beta$ value for the optically-thin regime excluding the last 3 epochs of our observations whose flux density values are beyond the expected values at this time. We also fit different $\mathrm{K}_{0}$ for each frequency which results in a $\beta=-1.23 \pm 0.01$. 
Figure 1: The multi-frequency light curve of SN 2008iz. The Merlin $5 \mathrm{GHz}$ data were obtained from Beswick et al. (2009), the urumqi $5 \mathrm{GHz}$ data from Marchili et al. (2010).

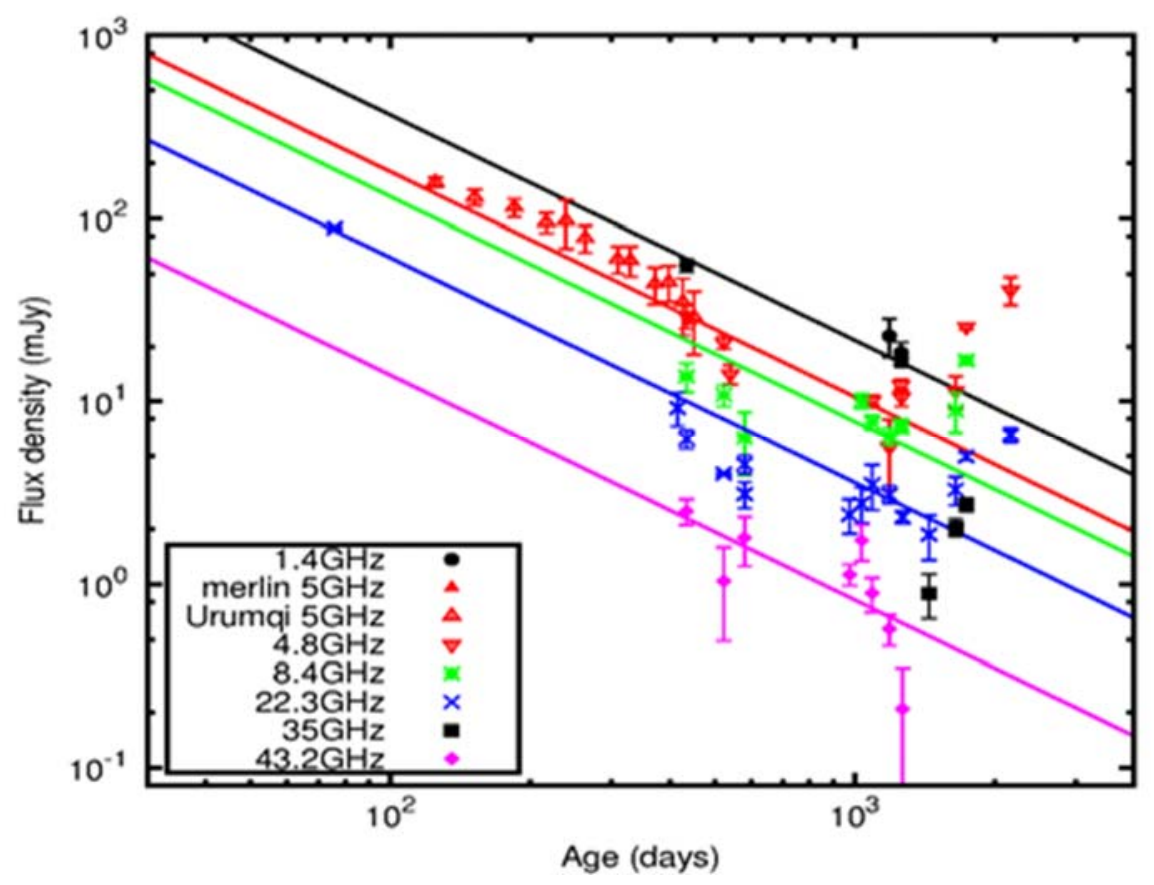

The values of $\mathrm{K}_{0}$ which appear to decrease with increasing frequency are $\mathrm{K}_{0(1.4 \mathrm{GHz})}=$ $(1.06 \pm 0.1) \times 10^{5}, \mathrm{~K}_{0(4.8 \mathrm{GHz})}=(5.19 \pm 0.6) \times 10^{4}, \mathrm{~K}_{0(8.4 \mathrm{GHz})}=(3.80 \pm 0.4) \times 10^{4}, \mathrm{~K}_{0(22.3 \mathrm{GHz})}$ $=(1.76 \pm 0.4) \times 10^{4}$ and $\mathrm{K}_{0(43.2 \mathrm{GHz})}=(4.02 \pm 0.6) \times 10^{3}$. Fig. 2 shows the normalized average spectrum of the variation of $\mathrm{K}_{0}$ with frequency in the optically thin regime. The sudden decrease of $\mathrm{K}_{0}$ value at $43.2 \mathrm{GHz}$ could be attributed to the supernova aging.

Figure 2: The normalized average spectrum of the variation of $K_{0}$ with frequency for the optically-thin regime.

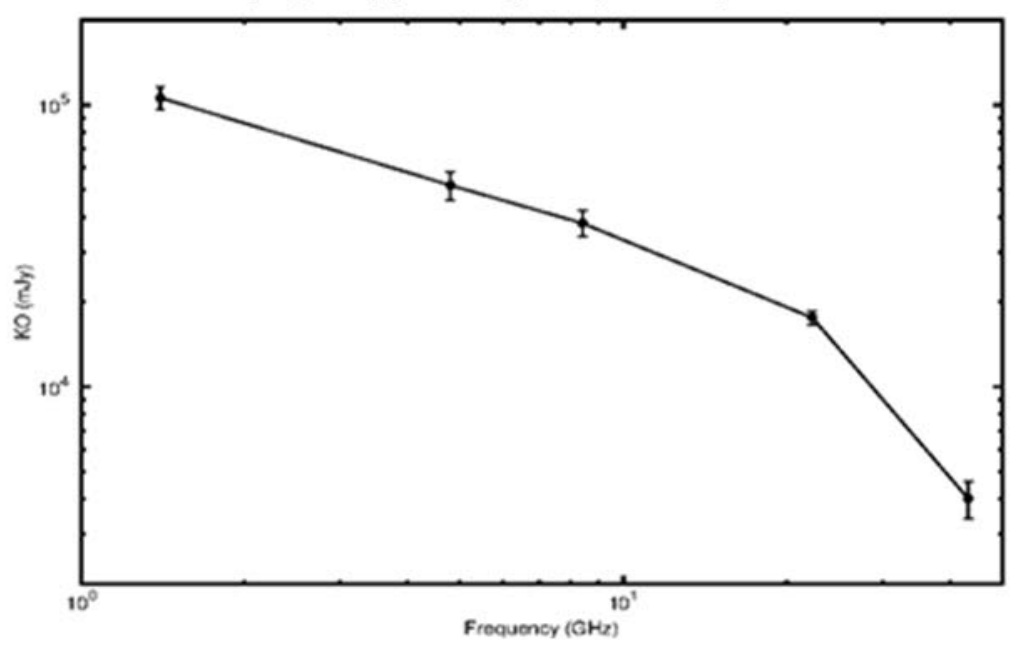


To fit the complete supernova light curve model from out data was impossible since we only have one $22.3 \mathrm{GHz}$ observation in the optically-thick regime as confirmed by Marchili et al (2010). We therefore made use of the best fit results of the simplified weiler model (equation 2) as submitted by Marchili et al. (2010) at $5 \mathrm{GHz}$. The model assumes the CSM distribution is homogeneous (i.e. there is no clumpy CSM) and do not consider SSA because it is insignificant. The fit parameters are $\mathrm{K}_{1}=(2.14 \pm 0.04) \times 10^{5} \mathrm{mJy}, \beta=-1.43 \pm 0.05$, $\mathrm{t}_{0}=18 \mathrm{Feb} 2008, \mathrm{~K}_{2}=$ $(11.0 \pm 0.7) \times 10^{5}, \delta=-2.65 \pm 0.10$ and from Brunthaler et al. (2010), we make use of the spectral index $\alpha=-1.08 \pm 0.08$. The simplified Weiler model fit to all frequencies at the same time is shown in Fig. 3 , with $\beta$ being a free parameter, yielding $\beta=-$ $1.41 \pm 0.02$.

$$
S_{(v)}=K_{I}\left(\frac{v}{5 \mathrm{GHz}}\right)^{\alpha}\left(\frac{t-t_{0}}{1 \text { day }}\right)^{\beta} e^{-\tau}
$$

where $\mathrm{K}_{1}$ is the flux density at $5 \mathrm{GHz}$ one day after the explosion, while $\alpha$ is the spectral index of the emission in the optically-thin regime. The parameters $\beta$, $t_{0}$ and $t$ are as indicated in equation 1 above, while $\tau$ is the opacity of the thermal electrons of the CSM to the radio emission which is modeled as

$$
\tau=K_{2}\left(\frac{v}{5 \mathrm{GHz}}\right)^{2.1}\left(\frac{t-t_{0}}{1 \text { day }}\right)^{\delta}
$$

where $\mathrm{K}_{2}$ is the uniform free-free absorption (FFA) value at $5 \mathrm{GHz}$ one day after the explosion, while $\delta$ is the absorption decline index related to the CSM radial density profile derived as $\delta=\alpha-\beta-3$. The exponent of -2.1 corresponds to the spectral dependence of FFA by thermally ionized gas in the radio regime.

The light curve indicates a flux enhancement at 1000 and 1700 days after the explosion (Fig. 4). The enhancement after day 1700 is detected at all frequencies and does not show signs of decline from the observations done so far. This indicates that the supernova shock-wave is encountering dense CSM which must be remnants of winds of SN 2008iz's progenitor star. 
Figure 3: The multi-frequency light curve of SN 2008iz. The Merlin $5 \mathrm{GHz}$ data were obtained from Beswick et al. (2009), the urumqi $5 \mathrm{GHz}$ data from Marchili et al. (2010).

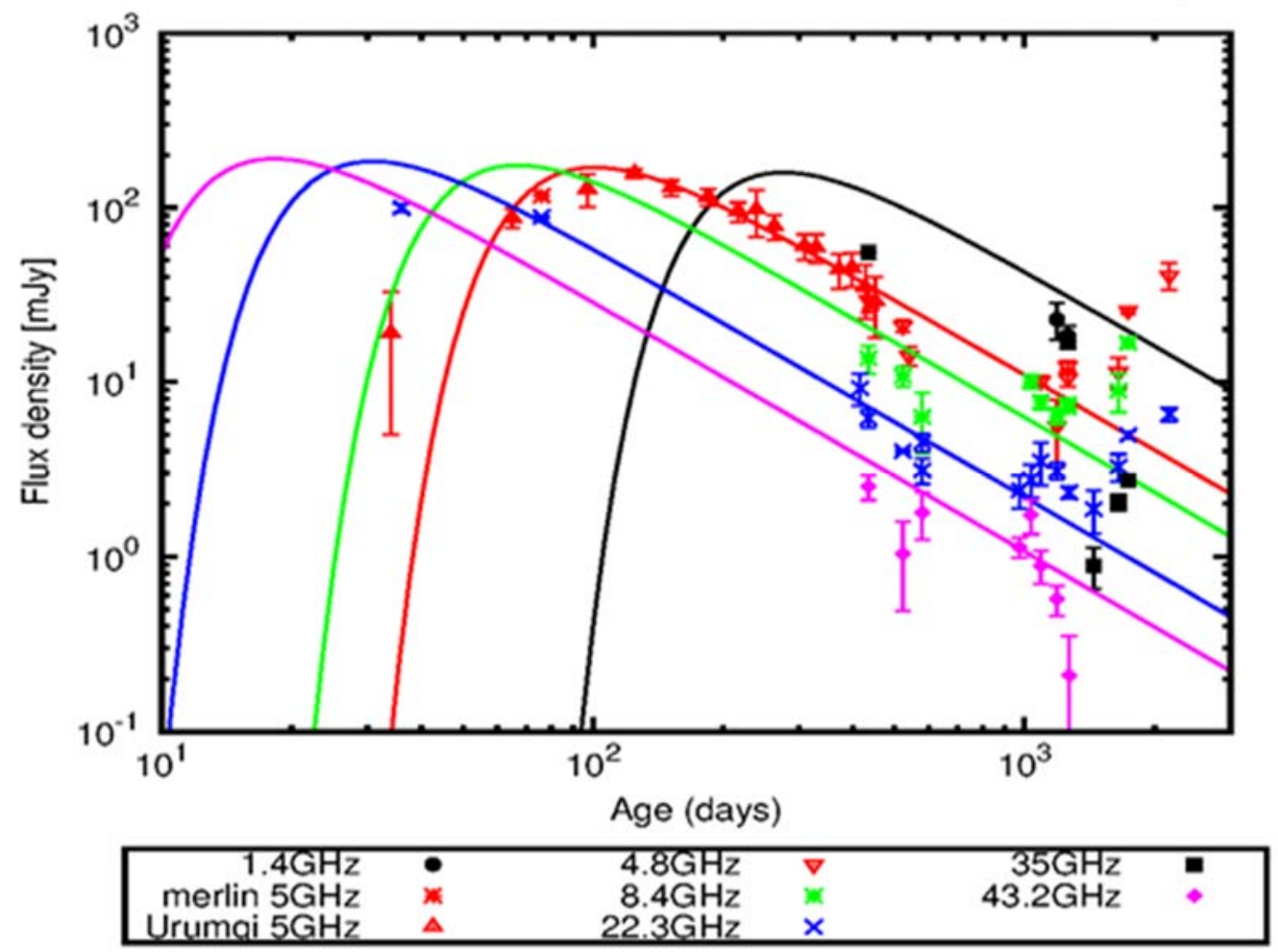

The power-law decline index of the light curve of SN 2008iz over the first 1000 days after the explosion is $\beta=-1.23 \pm 0.01$ and $-1.41 \pm 0.02$ for the two models fitted above. This value is steeper than that derived for SN 1993J $(\beta=-0.93)$ due to different processes in the supernovae, such as SSA being important in modelling SN 1993J light curve, but not in SN 2008iz (e.g. Weiler et al. 2002, Weiler et al. 2010). Montes et al (1998) also find a much flatter $\beta$ value of -0.73 for SN 1980K, with an inclusion of SSA in their model. However, supernova SN 1986J was reported to have a power-law index $\beta=-1.18$ (Weiler et al., 1990), with SSA reported not to be significant in this supernova, making it a better comparison for SN 2008iz. 
Figure 4: A zoom into our best sampled 22.3 GHz light curve between days 970 to 2200. The flux density enhancement at 1700 days was detected in both VLA and VLBA results

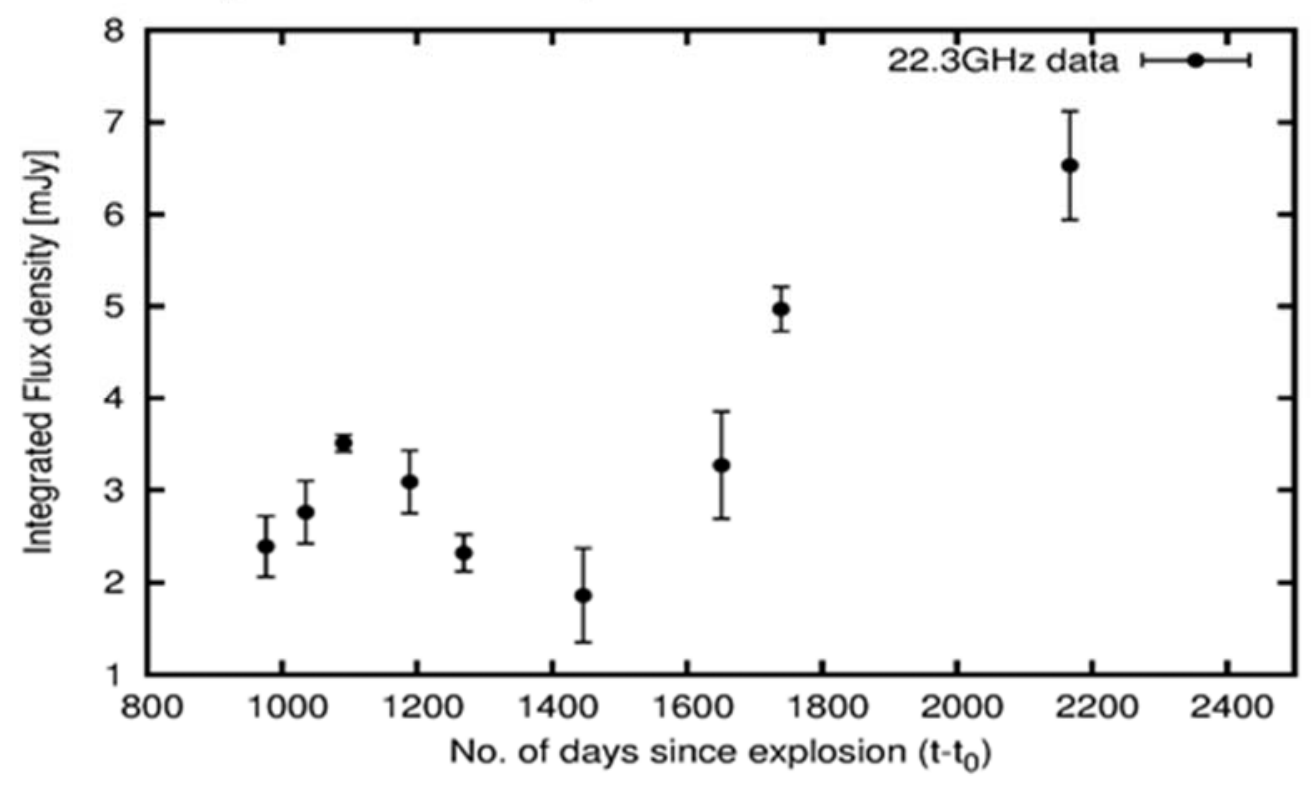

\section{Summary}

We report on multi-frequency VLA radio datasets for an on-going monitoring campaign of SN 2008iz. We fit two models to the data, a simple power-law and a simplified Weiler model, yielding

$\beta=-1.23 \pm 0.01$ and $-1.41 \pm 0.02$ respectively. The derived steep $\beta$ values as the supernova shock wave interacts with CSM indicate a significant drop in the circumstellar medium density. The light curve also uncovers a flux density enhancements at $\sim 1000$ and $\sim 1700$ days since the explosion. The observed flux density increase is attributed to an increase in the number density of shocked CSM electrons as the expanding shock wave encounters a clumpy denser medium. The medium is expected to be the remnant of winds of SN 2008iz's progenitor star. Therefore, a continued multiwavelength observation of SN 2008iz will offer more information on the nature of the dense circumstellar medium and also give an indications on the behaviour of its progenitor star.

Acknowledgement: $N$. Kimani was supported for this research through a stipend from the International Max Planck Research School (IMPRS) for Astronomy and Astrophysics at the Universities of Bonn and Cologne. 


\section{References}

Beswick, R. J., Muxlow, T. W. B., Pedlar, A., et al. (2009). Observations of the bright radio transient in M82: a new radio supernova? The Astronomer's Telegram, 2060

Bietenholz, M. F., Bartel, N., Rupen, M. P. (2010). Supernova 1986J Very Long Baseline Interferometry. II. The Evolution of the Shell and the Central Source, The Astrophysical Journal (ApJ), 712,1057

Brunthaler, A., Menten, K. M., Reid, M. J., et al. (2009a). Observations of the new transient radio source in the central region of M82, The Astronomer's Telegram, 2020

Brunthaler, A., Menten, K. M., Reid, M. J., et al. (2009b). Discovery of a bright radio transient in M82: a new radio supernova? Astronomy and Astrophysics $(A \& A), 499, \mathrm{~L} 17$

Brunthaler, A., Martí-Vidal, I., Menten, K. M., et al. (2010). VLBI observations of SN2008iz. I. Expansion velocity and limits on anisotropic expansion, Astronomy and Astrophysics (A\&A), 516, A27

Fraser, M., Smartt, S. J., Crockett, M., et al. (2009). Gemini-NIRI K-band observations of the sites of two recent radio transients in M82, The Astronomer's Telegram, 2131

Freedman, W. L., Hughes, S. M., Madore, B. F., et al. (1994). The Hubble Space Telescope Extragalactic Distance Scale Key Project. 1: The discovery of Cepheids and a new distance to M81, The Astrophysical Journal (ApJ), 427, 628

Marcaide, J. M., Alberdi, A., Ros, E., et al. (1997). Limit to the radio emission from a putative central compact source in SN1993J, The Astrophysical Journal (ApJ), 486, 31

Marchili, N., Martí-Vidal, I., Brunthaler, A., et al. (2010). The radio lightcurve of SN2008iz in M82 revealed by Urumqi observations, Astronomy and Astrophysics (A\&A), 509, A47

Martí-Vidal, I., Marcaide, J. M., Alberdi, A., et al. (2007). $8.4 \mathrm{GHz}$ VLBI observations of SN2004et in NGC6946, Astronomy and Astrophysics (A\&A), 470,1071 
Martí-Vidal, I., Marcaide, J. M., Alberdi, A., et al. (2011a). Radio emission of SN 1993J: the complete picture. I. Re-analysis of all the available VLBI data, Astronomy and Astrophysics (A\&A), 526, A142

Martí-Vidal, I., Marcaide, J. M., Alberdi, A., et al. (2011b). Radio emission of SN 1993J: the complete picture. II. Simultaneous fit of expansion and radio light curves, Astronomy and Astrophysics (A\&A), 526, A143

Montes, M. J., Van Dyk, S. D., Weiler, K. W., et al. (1998). Radio Observations of SN 1980K: Evidence for Rapid Presupernova Evolution, The Astrophysical Journal (ApJ), 506, 874

Muxlow, T. W. B., Beswick, R. J., Pedlar, A., et al. (2009). Discovery of a new transient radio source in the central region of M82, The Astronomer's Telegram, 2073

Weiler, K. W., Panagia, N., \& Sramek, R. A. (1990). Radio emission from supernovae. II - SN1986J: A different kind of type II, The Astrophysical Journal (ApJ), 364, 611

Weiler, K. W., Panagia, N., Montes, M. J., \& Sramek, R. A. (2002). Radio Emission from Supernovae and Gamma-Ray Bursters, Annual Review of Astronomy and Astrophysics (ARA\&A), 40, 387

Weiler, K. W., Williams, C. L., Panagia, N., et al. (2007). Long-Term Radio Monitoring of SN 1993J, The Astrophysical Journal (ApJ), 671, 1959

Weiler, K. W., Panagia, N., Sramek, R. A., et al. (2010). Radio emission from supernova, Memorie della Società Astronomia Italiana (Mem. S.A.lt), 81,374

Weiß, A., Neininger, N., Hüttemeister, S., \& Klein, U. (2001). The effect of violent star formation on the state of the molecular gas in M82, Astronomy and Astrophysics (A\&A), 365, 571 